\title{
AN EQUIVARIANT INDEX FORMULA IN CONTACT GEOMETRY
}

\author{
SEAN FitzPATRick
}

\begin{abstract}
Given an elliptic action of a compact Lie group $G$ on a co-oriented contact manifold $(M, E)$ one obtains two naturally associated objects: A $G$-transversally elliptic operator $\not_{b}$, and an equivariant differential form with generalized coefficients $\mathcal{J}(E, X)$ defined in terms of a choice of contact form on $M$.

We explain how the form $\mathcal{J}(E, X)$ is natural with respect to the contact structure, and give a formula for the equivariant index of $\emptyset_{b}$ involving $\mathcal{J}(E, X)$. A key tool is the Chern character with compact support developed by Paradan-Vergne [11, 12].
\end{abstract}

\section{Introduction}

Let $(M, E)$ be a compact contact manifold. Suppose that the contact distribution $E$ is co-oriented, and let $\alpha \in \mathcal{A}^{1}(M)$ be a contact form compatible with the coorientation. The distribution $E$ is contact if and only if the restriction of $d \alpha$ to $E$ is symplectic.

We equip $E$ with a complex structure $J$ compatible with $d \alpha$, defining an almostCauchy-Riemann (CR) structure $E_{1,0} \subset T M \otimes \mathbb{C}$ on $M$ whose underlying real subbundle is $E$ (see [5]). We suppose $E^{0,1}=\left(\overline{E_{1,0}}\right)^{*}$ is equipped with a compatible Hermitian metric $h$ and connection $\nabla$, and note the isomorphism $\psi: E^{*} \rightarrow E^{0,1}$ given in Section 3, equation (7) below.

Analogous to the definitions in [2], let $C(E)$ be the bundle of Clifford algebras over $M$ whose fibre at $x \in M$ is the Clifford algebra of $E_{x}^{*}$ with respect to the Riemannian metric on $E$ obtained from $h$. Then $\mathcal{E}=\bigwedge E^{0,1}$ is a spinor module for $C(E)$, with Clifford multiplication given for $\nu \in E_{x}$ by

$$
\mathbf{c}(\nu)=\iota(\psi(\nu))-\epsilon(\psi(\nu)) \text {. }
$$

Using the connection $\nabla$ and the Clifford multiplication (1) we can define a $\operatorname{Spin}^{c}$ Dirac-like operator $\emptyset_{b}$ whose principal symbol is $\sigma_{b}(x, \xi)=-i \mathbf{c}(q(\xi))$, where $(x, \xi) \in$ $T^{*} M$, and $q: T^{*} M \rightarrow E^{*}$ denotes projection. Since $\left(\sigma_{b}\right)^{2}(x, \xi)=\|q(\xi)\|^{2}$, the support of $\sigma_{b}$ is the annihilator bundle $E^{0}$ of $E$, whence $\emptyset_{b}$ is not elliptic.

Suppose now that a compact Lie group $G$ acts on $M$, such that the action preserves the contact distribution $E$, as well as its co-orientation, and choose $\alpha, J, h$ and $\nabla$ to be $G$-invariant. We require the action to be elliptic, meaning that $T M$ is spanned by $E$ and the vectors tangent to the $G$-orbits, or equivalently, that $E^{0}$ is transverse to the space $T_{G}^{*} M$ of covectors orthogonal to the $G$-orbits.

Thus, although $\emptyset_{b}$ is not elliptic, the requirement of ellipticity on the group action gives $\operatorname{Supp}\left(\sigma_{b}\right) \cap T_{G}^{*} M \subset M \times\{0\}$, which implies that $\emptyset_{b}$ is a $G$-transversally elliptic operator in the sense of Atiyah [1], and that the principal symbol $\sigma_{b}$ is a $G$-transversally elliptic symbol in the sense of Berline-Vergne [3].

Received by the editors January 23, 2008. 
Atiyah [1] has shown that the kernel and cokernel of any $G$-transversally elliptic operator $P$ will define trace-class representations of $G$, and that the principal symbol of $P$ defines an element in the equivariant $K$-theory $K_{G}\left(T_{G}^{*} M\right)$. The $G$-equivariant index of $P$ is well-defined, but only as a generalized function on $G$, given by the formula $[1,3]$ :

$$
\operatorname{index}^{G}(P)(g)=\operatorname{Tr}(g, \operatorname{ker} P)-\operatorname{Tr}\left(g, \operatorname{ker} P^{*}\right) .
$$

Berline and Vergne $[3,4]$ have given a character formula which gives the germ of (2) at $g \in G$ in terms of the integral over $T^{*} M(g)$ of certain equivariant differential forms, as follows:

For a $G$-transversally elliptic symbol $\sigma$, we have, for $g \in G$ and $X \in \mathfrak{g}(g)$ sufficiently small,

$$
\operatorname{index}^{G}(\sigma)\left(g e^{X}\right)=\int_{T^{*} M(g)}(2 i \pi)^{-\operatorname{dim} M(g)} \frac{\operatorname{Ch}_{B V}^{g}(\sigma, X) \hat{A}^{2}(M(g), X)}{D_{\mathbb{R}}(N(g), X)},
$$

where $\operatorname{Ch}_{B V}^{g}(\sigma, X)$ is the Chern character of [3]. For a $G$-transversally elliptic operator $P$ with principal symbol $\sigma(P)$, the equality of generalized functions $\operatorname{index}^{G}(P)=\operatorname{index}^{G}(\sigma(P))$ was proved in [4].

Recent work of Paradan and Vergne [12] allows one to replace the non-compactly supported equivariant forms in the integrand of (3) by forms with compact support, provided one passes to equivariant differential forms with generalized coefficients: these are $C^{-\infty}$ maps from $\mathfrak{g}$ to $\mathcal{A}(M)$, as in [8]. The space of all such forms will be denoted by $\mathcal{A}^{-\infty}(\mathfrak{g}, M)$.

When one allows generalized coefficients, it is possible to define a natural differential form on $M$ adapted to the contact structure as follows:

Let $\alpha$ be a contact form on $M$, let $D=d-\iota(X)$ be the equivariant differential, and let $\delta_{0}$ be the Dirac delta distribution on $\mathbb{R}$. Then we may define the form

$$
\mathcal{J}(E, X)=\alpha \wedge \delta_{0}(D \alpha(X)),
$$

which is well-defined as an element of $\mathcal{A}^{-\infty}(\mathfrak{g}, M)$.

Moreover, using the properties of the delta distribution, one has that

(1) $D \mathcal{J}(E, X)=0$, so that $\mathcal{J}(E, X)$ defines a class in $\mathcal{H}^{-\infty}(\mathfrak{g}, M)$, the equivariant cohomology of $M$ with generalized coefficients.

(2) $\mathcal{J}(E, X)$ is independent of the choice of contact form $\alpha$ and thus depends only on the contact structure $E$.

For a fixed $g \in G$, let $i: M(g) \rightarrow M$ denote the inclusion of the set of $g$-fixed points in $M$. In Proposition 2.7, we show that $(M(g), E(g))$ is again a contact manifold, with contact form $\alpha^{g}=i^{*} \alpha$, so that $\mathcal{J}(E(g), X)=\alpha^{g} \wedge \delta_{0}\left(D \alpha^{g}(X)\right)$ is again well defined, for $X \in \mathfrak{g}(g) \subset \mathfrak{g}$.

In this article, our interest in the form $\mathcal{J}(E, X)$ is due to its appearance in our formula for the equivariant index of the $G$-transversally elliptic operator $\square_{b}$. The results of $[11,12]$ allow us to re-write the integrand of (3) in terms of a Chern character $\mathrm{Ch}_{M Q}(\sigma, X)$ with "Gaussian shape" along the fibres of $E^{*}$ in the sense of [10], and a differential form $P_{\lambda}(X)$ with generalized coefficients whose support intersects $E^{0}$ in a compact set. We are then able to integrate over the fibres of $T^{*} M(g)$ to obtain: 
Theorem 1.1. Let $(M, E)$ be a compact, co-oriented contact manifold, and let $G$ be a compact Lie group acting elliptically on $M$. The G-equivariant index of $\emptyset_{b}$ is the generalized function on $G$ whose germ at $g \in G$ is given, for $X \in \mathfrak{g}(g)$ sufficiently small, by

$$
\operatorname{index}^{G}\left(D_{b}\right)\left(g e^{X}\right)=\int_{M(g)}(2 \pi i)^{-k(g)} \frac{\operatorname{Td}(E(g), X) \mathcal{J}(E(g), X)}{D_{\mathbb{C}}(N(g), X)},
$$

where $\operatorname{dim}(M(g))=2 k(g)+1$.

In particular, we have the following formula at the identity:

Theorem 1.2. For $X \in \mathfrak{g}$ sufficiently small,

$$
\operatorname{index}^{G}\left(D_{b}\right)\left(e^{X}\right)=\frac{1}{(2 \pi i)^{n}} \int_{M} \operatorname{Td}(E, X) \mathcal{J}(E, X) \text {. }
$$

In the case of an elliptic circle action on $M$, we can relate our index formula to the index of a Dirac operator on the quotient: Suppose that $M$ is a prequantum $U(1)$ bundle over a prequantizable Hamiltonian $G$-space $(B, \omega, \Phi)$ in the sense of [7]. If $\tilde{\alpha}$ is a connection form satisfying the prequantization condition $i D \tilde{\alpha}(X)=\pi^{*}(\omega-\Phi(X))$, then $\alpha=i \tilde{\alpha}$ is a contact form on $M$, and the action of $G \times U(1)$ on $M$ obtained from the action of $G$ on $B$ and the principal $U(1)$-action is elliptic.

Corollary 1.3. Let $\emptyset$ denote the Dolbeault-Dirac operator on sections of $\bigwedge T^{0,1} B$, let $\mathbb{L}=M \times_{U(1)} \mathbb{C}$, and denote $\sigma_{m}=\sigma(\not) \otimes \operatorname{Id}_{\mathbb{L} \otimes m}$. Then we have the following equality of generalized functions on $G \times U(1)$ :

$$
\operatorname{index}^{G \times U(1)}\left(\emptyset_{b}\right)(g, u)=\sum_{m \in \mathbb{Z}} u^{-m} \operatorname{index}^{G}\left(\sigma_{m}\right)(g) .
$$

\section{Elliptic actions on contact manifolds}

Let $G$ be a compact Lie group, and let $M$ be a $G$-manifold. We make use of the following notation:

Definition 2.1. The set $\bigcup_{x \in M}\left(T_{x}(G \cdot x)\right)^{0} \subset T^{*} M$ of covectors orthogonal to the $G$-orbits will be denoted $T_{G}^{*} M$.

Definition 2.2. Let $\eta \in \mathcal{A}^{1}(M)$ be an invariant 1-form on a $G$-manifold $M$. We define the $\boldsymbol{\eta}$-moment map to be the map $f_{\eta}: M \rightarrow \mathfrak{g}^{*}$ given by the pairing

$$
<f_{\eta}(m), X>=\eta_{m}\left(X_{M}(m)\right),
$$

for any $X \in \mathfrak{g}$, where $X_{M}$ is the vector field on $M$ corresponding to $X$ via the infinitesimal action of $\mathfrak{g}$ on $M$.

We denote by $C_{\eta}$ the zero-level set $f_{\eta}^{-1}(0) \subset M$ of the $\eta$-moment map.

For any $G$-space $V$, we will denote by $V(g)$ the subset of $V$ fixed by the action of an element $g \in G$. 
Remark 2.3. Let $\theta$ be the canonical 1-form on $T^{*} M$, and consider the lift of the action of $G$ on $M$ to $T^{*} M$. This action is Hamiltonian, and $f_{\theta}: T^{*} M \rightarrow \mathfrak{g}^{*}$ is the corresponding moment map. We may describe the space $T_{G}^{*} M$ according to $T_{G}^{*} M=C_{\theta}$.

Let $(M, E)$ denote a compact co-oriented contact manifold, and let $G$ be a compact Lie group acting on $M$ preserving the contact structure $E$, and the co-orientation. Choose a $G$-invariant contact form $\alpha$ compatible with the co-orientation. That is, if we let $E_{+}^{0}$ be the connected component of $E^{0} \backslash 0$ that is positive with respect to the co-orientation, then $\alpha(M) \subset E_{+}^{0}$. We will suppose such a choice of contact form has been fixed throughout the article. ${ }^{1}$

Remark 2.4. The space $E_{+}^{0} \subset T^{*} M$ is a symplectic cone over the base $M$, called the symplectization of $M$. The symplectic form on $E_{+}^{0}$ is the pullback under inclusion of the canonical symplectic form on $T^{*} M$. The cotangent lift of an action of $G$ on $M$ preserving $E$ restricts to a symplectic action of $G$ on $E_{+}^{0}$ commuting with the natural $\mathbb{R}_{+}$action.

Definition 2.5. The action of $G$ on $(M, E)$ is said to be elliptic if and only if $T_{G}^{*} M \cap E^{0}=0$.

For the remainder of this article, we will impose this stronger condition on the action of $G$ on $M$.

Remark 2.6. The action of $G$ on $(M, E)$ is elliptic if the orbits of $G$ in $M$ are nowhere tangent to the contact distribution. Alternatively, if $\Phi: E_{+}^{0} \rightarrow \mathfrak{g}^{*}$ is the restriction of $f_{\theta}$ to $E_{+}^{0}$, then the action is elliptic if and only if zero is not in the image of $\Phi$.

Associated to the chosen contact form $\alpha$ is the Reeb vector field, which is the vector field $Y \in \Gamma(T M)$ such that

$$
\iota(Y) \alpha=1 \text { and } \iota(Y) d \alpha=0 .
$$

Accordingly, we obtain a splitting $T M=E \oplus \mathbb{R} Y$, dual to the splitting $T^{*} M=$ $E^{*} \oplus \mathbb{R} \alpha$ given by the choice of contact form. (5):

The following proposition is a key lemma for our proof of the fixed-point formula

Proposition 2.7. Let $(M, \alpha)$ be a co-oriented contact manifold, and suppose $G$ is a compact group acting on $M$ elliptically. For any $g \in G$, let $i: M(g) \rightarrow M$ denote inclusion of the g-fixed points. Then we have:

(1) The submanifold $M(g) \subset M$ is a contact manifold, and $\alpha^{g}=i^{*} \alpha$ is a contact form on $M(g)$.

(2) The action of the centralizer $G(g)$ of $g$ in $G$ on $M(g)$ is elliptic.

Proof. (1) Let $T M=E \oplus \mathbb{R} Y$, where $Y$ is the Reeb vector field associated to the $G$-invariant contact form $\alpha$, and thus $G$-invariant as well.

Denote by $N(g)$ the normal bundle to $M(g)$ in $M$. Then we have that

$$
\left.T M\right|_{M(g)}=T M(g) \oplus N(g)=E(g) \oplus \mathbb{R} Y^{g} \oplus N(g)
$$

\footnotetext{
${ }^{1} \mathrm{~A}$ good exposition of this terminology can be found in [9].
} 
by the invariance of $Y$, where $Y^{g}=\left.Y\right|_{M(g)}$ and $E(g)$ is the subset of $E$ fixed by the action of $g \in G$.

Choose any $m \in M(g)$. Then we know that $\left.d \alpha\right|_{E_{m}}$ is symplectic. Moreover, the action of $G$ on the symplectic vector space $E_{m}$ preserves $\alpha$, and thus the symplectic structure, whence $E_{m}(g)$ is symplectic, with symplectic form $d \alpha^{g}=i^{*} d \alpha$.

Finally, since $\alpha(M(g)) \subset T^{*} M(g)=(T M(g))^{*}$, and $\iota\left(Y^{g}\right) \alpha^{g}=1$, we have $\operatorname{ker}\left(\alpha^{g}\right) \cap \mathbb{R} Y^{g}=0$, whence $\operatorname{ker}\left(\alpha^{g}\right) \subset E(g)$, and a dimension count gives $\operatorname{ker}\left(\alpha^{g}\right)=$ $E(g)$.

(2) Recall that the action of $G$ on $M$ is elliptic if and only if zero is not in the image of the moment map $\Phi: E_{+}^{0} \rightarrow \mathfrak{g}^{*}$ (Remark 2.6). Let $H=G(g)$, and let $\mathfrak{h}$ be the Lie algebra of $H$.

If $x \in E_{+}^{0}(g)$, we have by the equivariance of $\Phi$ that $\Phi(x) \in \mathfrak{h}^{*}$. Thus, the corresponding moment map $\Psi: E_{+}^{0}(g) \rightarrow \mathfrak{h}^{*}$ for the action of $G(g)$ on $E_{+}^{0}(g)$ is simply the restriction of $\Phi$ to $E_{+}^{0}$ (the projection from $\mathfrak{g}^{*}$ to $\mathfrak{h}^{*}$ being redundant).

Since $\Psi=\left.\Phi\right|_{E_{+}^{0}(g)}$, it follows that zero is not in the image of $\Psi$, and thus the action of $G(g)$ on $M(g)$ is elliptic.

\section{Definition of the operator $\not_{b}$}

Let $\alpha \in \Gamma\left(E^{0} \backslash 0\right)$ be a given choice of $G$-invariant contact form on $(M, E)$. Then $\left.d \alpha\right|_{E}$ defines a symplectic structure on the fibres of $E$, so that $E$ is a symplectic vector bundle over $M$. Let $J$ be a $G$-invariant complex structure on $E$.

If $\beta$ is any other contact form, then $\beta=e^{f} \alpha$ for some $f \in C^{\infty}(M)$. Thus we have $d \beta=e^{f} d f \wedge \alpha+e^{f} d \alpha$, whence $\left.d \beta\right|_{E}=\left.e^{f} d \alpha\right|_{E}$. Therefore, if $J$ is a complex structure on $E$ compatible with $d \alpha$, it is also compatible with $d \beta$, and thus depends only on the contact structure $E$.

The pair $(E, J)$ determines an almost-CR structure $E_{1,0}$ on $M$ whose underlying real bundle is the contact distribution (see [5]). Thus $E_{1,0} \cap \overline{E_{1,0}}=0$, and so $E_{1,0} \oplus E_{0,1}=E \otimes \mathbb{C}$, where $E_{0,1}=\overline{E_{1,0}}$. Let $E^{1,0}=\left(E_{1,0}\right)^{*}$, giving the $G$-invariant decomposition

$$
E^{*} \otimes \mathbb{C}=E^{1,0} \oplus E^{0,1}
$$

into $\pm i$-eigenspaces of $J$, where for $\eta \in T^{*} M$ and $\xi \in T M$ the induced almost-complex structure on $E^{*}$ is given by $J(\eta)(\xi)=\eta(J(\xi))$

Let $\psi: E^{*} \stackrel{\simeq}{\longrightarrow} E^{0,1}$ be the isomorphism given by

$$
\psi(\eta)=\eta+i J(\eta) .
$$

Let $p: E^{*} \rightarrow M, q: T^{*} M \rightarrow E^{*}$, and $\pi_{M}=p \circ q: T^{*} M \rightarrow M$ denote projections. Let $h$ be the $G$-invariant Hermitian metric on $E^{0,1}$ determined by $J$ and $d \alpha$, and let $\nabla$ be a $G$-invariant Hermitian connection on $E^{0,1}$. The metric $h$ determines an invariant Riemannian metric on $E$. Let $C(E) \rightarrow M$ be the bundle whose fibre over $x \in M$ is the Clifford algebra of $E_{x}$ with respect to this metric. For any $\nu \in E_{x}$, we have the Clifford multiplication given by

$$
\mathbf{c}(\nu)=\iota(\psi(\nu))-\epsilon(\psi(\nu)),
$$

where $\epsilon(\eta)$ denotes exterior multiplication by $\eta$, and $\iota(\eta)$ denotes contraction with respect to $h: \iota(\eta, \xi)=h(\eta, \xi)$. The multiplication c makes $\mathcal{E}=\bigwedge E^{0,1}$ into a spinor module for $C(E)$. 
Using $\nabla$ and $\mathbf{c}$, we define a $G$-invariant differential operator $\not_{b}: \Gamma(\mathcal{E}) \rightarrow \Gamma(\mathcal{E})$ by the composition

$$
\Gamma(\mathcal{E}) \stackrel{\nabla}{\longrightarrow} \Gamma\left(T^{*} M \otimes \mathcal{E}\right) \stackrel{q}{\rightarrow} \Gamma\left(E^{*} \otimes \mathcal{E}\right) \stackrel{\mathbf{c}}{\rightarrow} \Gamma(\mathcal{E}) .
$$

In the case that $E_{1,0}$ is integrable, $M$ is a Cauchy-Riemann manifold, and we can choose $\nabla$ such that

$$
\not_{b}=\sqrt{2}\left(\bar{\partial}_{b}+\bar{\partial}_{b}^{*}\right)
$$

where $\bar{\partial}_{b}$ is the tangential Cauchy-Riemann operator defined with respect to $E_{1,0}$ [5].

Write $\mathcal{E}=\mathcal{E}^{+} \oplus \mathcal{E}^{-}$with respect to the $\mathbb{Z}_{2}$-grading given by exterior degree, and let $\sigma_{b}: \pi_{M}^{*} \mathcal{E}^{+} \rightarrow \pi_{M}^{*} \mathcal{E}^{-}$denote the principal symbol of $\emptyset_{b}$, given for any $(x, \xi) \in T^{*} M$ and $\gamma \in \mathcal{E}^{+}$by

$$
\sigma_{b}(x, \xi)(\gamma)=-i \mathbf{c}(q(\xi)) \gamma
$$

For any morphism $\sigma$ on $\pi_{M}^{*} \mathcal{E}$, define

$$
\operatorname{Supp}(\sigma)=\left\{(x, \xi) \in T^{*} M \mid \sigma(x, \xi) \text { is not invertible }\right\} .
$$

Since $\sigma_{b}^{2}(x, \xi)=\|q(\xi)\|^{2}$, we have $\operatorname{Supp}\left(\sigma_{b}\right)=E^{0}$. This implies that for an elliptic $G$-action on $M, \not_{b}$ is a $G$-transversally elliptic differential operator in the sense of Atiyah [1], since $E^{0} \cap T_{G}^{*} M=0$.

Therefore, $\left[\pi_{M}^{*} \mathcal{E}, \sigma_{b}\right]$ defines an equivariant $K$-theory class in $K_{G}\left(T_{G}^{*} M\right)$. This class is independent of the almost-CR structure (since any two such structures with underlying real bundle $E$ are homotopic) and the Hermitian metric. A formula for the equivariant index of this class has been given by Berline and Vergne [3, 4], but requires the integration of non-compactly supported forms on $T^{*} M$.

Following Paradan and Vergne [12], we will instead pass to equivariant differential forms with generalized coefficients, which will allow us to construct a compactly supported form whose integral over $T^{*} M$ agrees with that of the Berline-Vergne formula, and for which the integral over the fibres is easily carried out.

Before dealing with the technical details of this construction and the proof of our main theorem, we pause to consider two simple examples in which our index theorem may be applied.

\section{Examples}

The simplest examples of an elliptic group action on a contact manifold involve free circle actions. A particularly simple example is discussed in [14]: that of a circle acting on itself by multiplication.

Example 1: $S^{1}$

Consider the circle $S^{1}=\left\{e^{i \theta} \mid \theta \in \mathbb{R}\right\}$. The form $d \theta$ is a contact form on $S^{1}$, with the zero section as the contact distribution. The group $U(1)=\left\{e^{i \phi}\right\}$ acts freely on $S^{1}$ by multiplication. The action is elliptic, since $T_{G}^{*} S^{1}=0\left(\right.$ while $\left.E^{0}=T^{*} S^{1}\right)$.

Here, our operator is $\emptyset_{b}=0$, and since $T_{G}^{*} S^{1}=0$, even the zero operator on $S^{1}$ is $U(1)$-transversally elliptic. The $U(1)$-equivariant index is given simply by

$$
\operatorname{index}^{G}(0)\left(e^{i \phi}\right)=\int_{S^{1}} \mathcal{J}(\phi)=2 \pi \delta_{0}(\phi)=\sum_{m \in \mathbb{Z}} e^{i m \phi},
$$


where the last equality is valid for $\phi$ sufficiently small, using the Poisson summation formula for $\delta_{0}$.

Example 2: $S^{3}$

Let $M=S^{3}$ be the unit sphere in $\mathbb{R}^{4}$ with co-ordinates $\left(x_{1}, y_{1}, x_{2}, y_{2}\right)$, and consider the frame $\{X, Y, T\}$ for $T S^{3}$ given by

$$
\begin{aligned}
X & =x_{2} \frac{\partial}{\partial x_{1}}-y_{2} \frac{\partial}{\partial y_{1}}-x_{1} \frac{\partial}{\partial x_{2}}+y_{1} \frac{\partial}{\partial y_{2}} \\
Y & =-y_{2} \frac{\partial}{\partial x_{1}}-x_{2} \frac{\partial}{\partial y_{1}}+y_{1} \frac{\partial}{\partial x_{2}}+x_{1} \frac{\partial}{\partial y_{2}} \\
T & =y_{1} \frac{\partial}{\partial x_{1}}-x_{1} \frac{\partial}{\partial y_{1}}+y_{2} \frac{\partial}{\partial x_{2}}-x_{2} \frac{\partial}{\partial y_{2}} .
\end{aligned}
$$

A contact structure is given by $E=T S^{3} / \mathbb{R} T$. If we let $\{\xi, \zeta, \alpha\}$ denote the corresponding co-frame, then $\alpha$ is a contact form on $S^{3}$. In co-ordinates we have

$$
\alpha=y_{1} d x_{1}-x_{1} d y_{1}+y_{2} d x_{2}-x_{2} d y_{2},
$$

and one readily sees that $\alpha(T)=x_{1}^{2}+y_{1}^{2}+x_{2}^{2}+y_{2}^{2}=1$, so that $T$ is the Reeb field for $\alpha$.

We let $U(1)$ act on $S^{3}$, with action given in complex co-ordinates as follows: identify $\mathbb{R}^{4} \cong \mathbb{C}^{2}$ via $z_{j}=x_{j}+i y_{j}, j=1,2$. The action of $e^{i \phi} \in U(1)$ on $\mathbb{C}^{2}$ by $e^{i \phi} \cdot\left(z_{1}, z_{2}\right)=$ $\left(e^{i \phi} z_{1}, e^{i \phi} z_{2}\right)$ restricts to an action of $U(1)$ on $S^{3}$. Let $\mathfrak{g}=i \mathbb{R}$ denote the Lie algebra of $G$, and note that the infinitesimal action of $\mathfrak{g}$ on $M$ is given by $i \phi \mapsto \phi T$. The orbits of the action are thus transverse to the contact distribution $E$, whence the action of $U(1)$ on $S^{3}$ is elliptic.

The almost-CR structure on $M$ is given by taking $E_{1,0}=\mathbb{C} Z$, where $Z=\frac{1}{\sqrt{2}}(X+$ $i Y)$. The corresponding covector in $E^{*} \otimes \mathbb{C}$ is $\theta=\frac{1}{\sqrt{2}}(\xi-i \zeta)$. The associated complex structure on $E$ comes from the complex structure on $\mathbb{C}^{2}$, and is given by $J(X)=-Y$ and $J(Y)=X$, so that $J(\xi)=\zeta$, and $J(\zeta)=-\xi$ on $E^{*}$. Since this structure is integrable, $M$ is a CR manifold, and $\emptyset_{b}=\sqrt{2}\left(\bar{\partial}_{b}+\bar{\partial}_{b}^{*}\right)$.

Writing $\eta \in T_{x}^{*} S^{3}$ as $\eta=a \xi+b \zeta+c \alpha$, the symbol of $\emptyset_{b}$ is given by

$$
\sigma_{b}(x, \eta)=-i \sqrt{2}((a+i b) \iota(\bar{Z})-(a-i b) \epsilon(\bar{\theta})),
$$

from which we see that $\sigma_{b}^{2}(x, \eta)=a^{2}+b^{2}$.

Finally, for $\phi$ sufficiently small, the $U(1)$-equivariant index of $\emptyset_{b}$ is given by

$$
\begin{aligned}
\operatorname{index}^{U(1)}\left(\not_{b}\right)\left(e^{i \phi}\right) & =\frac{1}{2 \pi i} \int_{S^{3}} \operatorname{Td}(E, \phi) \mathcal{J}(E, \phi)=2 \pi\left(\delta_{0}(\phi)-i \delta_{0}^{\prime}(\phi)\right) \\
& =\sum_{m \in \mathbb{Z}}(1-m) e^{i m \phi}=\sum_{m \in \mathbb{Z}} \frac{1}{2 \pi i} \int_{S^{2}} \operatorname{Td}\left(S^{2}\right) e^{-i m(\omega-\phi)},
\end{aligned}
$$

since

$$
\mathcal{J}(E, \phi)=\alpha \wedge \delta_{0}(d \alpha-\phi)=\alpha\left(\delta_{0}(-\phi)+\delta_{0}^{\prime}(-\phi) d \alpha\right)
$$


while, if $\pi: S^{3} \rightarrow S^{2}$ denotes the projection onto the orbit space, then

$$
\begin{aligned}
\operatorname{Td}(E, \phi) & =\operatorname{Td}\left(\pi^{*} T S^{2}, \phi\right)=\pi^{*} \operatorname{Td}\left(S^{2}\right) \\
& =\pi^{*}(1+i \omega)=1+i d \alpha .
\end{aligned}
$$

Note that the last equality in (11) is an instance of Corollary 6.6.

\section{Equivariant differential forms with generalized coefficients}

Let $N$ be a smooth manifold, not necessarily compact. Let $\mathcal{A}^{\infty}(\mathfrak{g}, N)$ denote the complex of smooth equivariant differential forms on $N$; that is, the space of smooth maps $\alpha: \mathfrak{g}=\operatorname{Lie}(G) \rightarrow \mathcal{A}(N)$ that commute with the actions of $G$ on $\mathfrak{g}$ and $N$.

The space $\mathcal{A}^{\infty}(\mathfrak{g}, N)$ is equipped with the equivariant differential $D$, given by

$$
(D \alpha)(X)=d(\alpha(X))-\iota\left(X_{N}\right) \alpha(X),
$$

for any $X \in \mathfrak{g}$, where $\iota\left(X_{N}\right)$ is contraction by the vector field on $N$ generated by the infinitesimal action of $X \in \mathfrak{g}$. We have $D^{2}=0$ on $\mathcal{A}^{\infty}(\mathfrak{g}, N)$, whence we can define the equivariant cohomology $\mathcal{H}^{\infty}(\mathfrak{g}, N)$.

We can define further the complex $\mathcal{A}^{-\infty}(\mathfrak{g}, N)$ of equivariant differential forms with generalized coefficients: this is the space of G-equivariant generalized functions from $\mathfrak{g}$ to $\mathcal{A}(N)$ [8]. Thus, for any $\alpha \in \mathcal{A}^{-\infty}(\mathfrak{g}, N)$ and any compactly supported test function $\phi \in C^{\infty}(\mathfrak{g}), \int_{\mathfrak{g}} \alpha(X) \phi(X) d X$ is a smooth differential form on $N$.

The equivariant differential $D$ extends to $\mathcal{A}^{-\infty}(\mathfrak{g}, N)$, with $D^{2}=0$, and so it is possible to define the space the space $\mathcal{H}^{-\infty}(\mathfrak{g}, N)$ of equivariant cohomology with generalized coefficients. See $[8,12,14]$ for examples where this space is computed.

For non-compact $N$, we may consider as well the cohomology of (generalized) equivariant differential forms with compact support on $N$, denoted by $\mathcal{H}_{c}^{ \pm \infty}(\mathfrak{g}, N)$.

5.1. Forms in $\mathcal{A}^{-\infty}(\mathfrak{g}, N)$ derived from distributions on $\mathbb{R}$. It will be useful in our computation of the equivariant index to work in terms of the following generalized functions on $\mathbb{R}$ :

Note that we have

$$
\delta_{+}(x)=\lim _{\epsilon \rightarrow 0} \frac{i}{x+i \epsilon}, \quad \delta_{-}(x)=\lim _{\epsilon \rightarrow 0} \frac{-i}{x-i \epsilon} .
$$

$$
\delta_{+}(x)+\delta_{-}(x)=\lim _{\epsilon \rightarrow 0} \frac{\epsilon}{x^{2}+\epsilon^{2}},
$$

which we identify as the Dirac delta distribution $\delta_{0}(x)$, giving the first of the following identities:

$$
\begin{gathered}
\delta_{+}+\delta_{-}=\delta_{0}, \\
-i x \delta_{+}(x)=i x \delta_{-}(x)=1, \quad x \delta_{0}(x)=0,
\end{gathered}
$$

and for any $a \in \mathbb{R} \backslash\{0\}$, we have

$$
a \delta_{0}(a x)=\left\{\begin{array}{ll}
\delta_{0}(x), & \text { if } a>0 \\
-\delta_{0}(x), & \text { if } a<0
\end{array}, \quad a \delta_{ \pm}(a x)=\left\{\begin{array}{ll}
\delta_{ \pm}(x) & \text { if } a>0 \\
-\delta_{\mp}(x) & \text { if } a<0
\end{array} .\right.\right.
$$

The integral representations of these generalized functions given by

$$
\delta_{+}(x)=\frac{1}{2 \pi} \int_{0}^{\infty} e^{i x t} d t, \quad \delta_{-}(x)=\frac{1}{2 \pi} \int_{-\infty}^{0} e^{i x t} d t
$$


will be helpful in the computation of the index formulas to follow.

Distributions on $\mathbb{R}$, such as the above, can be used to define equivariant differential forms with generalized coefficients. For example, given an invariant 1-form $\beta$ on $N$, the form $\delta_{0}(D \beta)$, which we may view as the oscillatory integral $\int_{-\infty}^{\infty} e^{-i t D \beta} d t$, is well-defined as a generalized equivariant form wherever the pairing $X \mapsto \beta\left(X_{N}\right)$ is non-zero.

Let us explain in general how such a form is well-defined: Let $u \in C^{-\infty}(\mathbb{R})$ be a distribution on $\mathbb{R}$. We may consider its pull-back by a smooth, proper map $h: \mathfrak{g} \rightarrow \mathbb{R}$, which will give a well-defined distribution $h^{*} u=u \circ h$ on $\mathfrak{g}$ provided

$$
h^{*}(W F(u)) \cap(\mathfrak{g} \times\{0\})=\emptyset \subset T^{*} \mathfrak{g},
$$

where $W F(u)$ denotes the wavefront set of $u[6]$.

Remark 5.1. Note that for the resulting distribution on $\mathfrak{g}$, we have $W F\left(h^{*} u\right) \subset$ $h^{*} W F(u)$. Furthermore, for any derivative $u^{(j)}$ of $u$, we have $W F\left(u^{(j)}\right) \subset W F(u)$, so that if a map $h$ satisfies the condition above with respect to $u$, it does so for all the derivatives of $u$ as well.

Now, if $\beta$ is an invariant 1-form on $M$, then for a fixed point $m \in M, f_{\beta}(m)$ gives us a linear map from $\mathfrak{g}$ to $\mathbb{R}$. If $f_{\beta}(m)$ satisfies (15) for all $m \in M$, then we may set

$$
u(D \beta)(X)=u\left(d \beta-f_{\beta}(X)\right)=\sum_{j} \frac{u^{(j)}\left(-f_{\beta}(X)\right)}{j !}(d \beta)^{j},
$$

which is well-defined by Remark 5.1

On a contact manifold $(M, E)$ on which a Lie group $G$ acts elliptically, consider the form

$$
\mathcal{J}(E, X)=\alpha \wedge \delta_{0}(D \alpha(X)),
$$

where $\alpha$ is any contact form.

The ellipticity hypothesis ensures that the pairing $X \mapsto \alpha\left(X_{M}\right)$ is non-zero: We have $W F\left(\delta_{0}\right)=\{0\} \times(\mathbb{R} \backslash 0)$, while $f_{\alpha}^{-1}(0)=\emptyset$. Thus for all $m \in M, \eta=f_{\alpha}(m)$ is non-zero, and

$$
\eta^{*}\left(W F\left(\delta_{0}\right)\right)=\left\{(X, t \eta) \in \mathfrak{g} \times \mathfrak{g}^{*} \mid(\eta(X), t) \in\{0\} \times(\mathbb{R} \backslash 0)\right\} .
$$

Since $t \eta$ is never zero, (15) is satisfied.

Using the properties of the delta distribution given above, we obtain the following:

Proposition 5.2. Let $(M, \alpha)$ be a co-oriented contact manifold on which a Lie group $G$ acts elliptically. Then the form $\mathcal{J}(E, X)$ is equivariantly closed, and independent of the choice of contact form.

Proof. We have:

$$
D\left(\alpha \wedge \delta_{0}(D \alpha)\right)=D \alpha \wedge \delta_{0}(D \alpha)=0 \text { by }(13),
$$

while if we change $\alpha$ to $e^{f} \alpha$ for some $f \in C^{\infty}(M)$ we have using (14) that

$$
\begin{aligned}
e^{f} \alpha \wedge \delta_{0}\left(D\left(e^{f} \alpha\right)\right) & =e^{f} \alpha \wedge \delta_{0}\left(e^{f}(d f \wedge \alpha+D \alpha)\right) \\
& =\alpha \wedge \delta_{0}(d f \wedge \alpha+D \alpha)=\alpha \wedge \delta_{0}(D \alpha),
\end{aligned}
$$

where in the last equality we have used (16), and the fact that $\alpha \wedge \alpha=0$. 
Remark 5.3. Recall [7] that given a symplectic manifold $(N, \omega)$ of dimension $2 \mathrm{n}$ and a Hamiltonian action of a Lie group $G$ on $N$ with moment map $\Psi$ we may define the Duistermaat-Heckman distribution $u_{D H}$ on $C_{c}^{\infty}\left(\mathfrak{g}^{*}\right)$ by

$$
<\phi, u_{D H}>=\int_{\mathfrak{g}^{*}} \phi u_{D H}=\frac{1}{(2 \pi)^{n}} \int_{N}\left(\Psi^{*} \phi\right) e^{\omega} .
$$

The Fourier transform of $u_{D H}$ is given, for $h \in C_{c}^{\infty}(\mathfrak{g})$ by

$$
<\widehat{u_{D H}}, h>=<u_{D H}, \hat{h}>=\int_{\mathfrak{g}} h(X) I(X) d X,
$$

so that $\widehat{u_{D H}}=I(X) d X$, where

$$
I(X)=\int_{\mathfrak{g} *} e^{-i<X, \xi>} u_{D H}(\xi)=\frac{1}{(2 \pi)^{n}} \int_{N} e^{-i<X, \Psi>} e^{\omega}=(2 \pi i)^{-n} \int_{N} e^{i \omega(X)} .
$$

Now, given a co-oriented contact manifold $(M, E)$ of dimension $2 n+1$, consider the annihilator $E^{0}$ of $E$. Although not quite a symplectic manifold, since the form $\omega=d(t \alpha)$ is degenerate for $t=0$, we have the moment map $\Psi=t f_{\alpha}$, and if we compute $I(X)$ in this case, we find

$$
I(X)=\frac{1}{(2 \pi i)^{n+1}} \int_{E^{0}} e^{i \omega(X)}=\frac{1}{(2 \pi i)^{n}} \int_{M} \mathcal{J}(E, X) .
$$

Similarly, on the symplectic manifold $E_{+}^{0}$ we obtain an expression for the Fourier transform of the Duistermaat-Heckman distribution by replacing $\delta_{0}$ by $\delta_{+}$.

5.2. Cohomology with support. We give here a quick summary of the material in [11] and [12] that is relevant to the proof of our index theorem.

Suppose $F$ is a closed, $G$-invariant subset of $N$. Then there are two cohomology spaces associated to $F$ defined in [11]: $\mathcal{H}^{\infty}(\mathfrak{g}, N, N \backslash F)$, the relative equivariant cohomology of $N$, and $\mathcal{H}_{F}^{\infty}(\mathfrak{g}, N)$, the equivariant cohomology with compact support in $N$.

Representatives of cohomology classes in the former are pairs $(\eta, \xi)$, where $\eta \in$ $\mathcal{A}^{\infty}(\mathfrak{g}, N)$ and $\xi \in \mathcal{A}^{\infty}(\mathfrak{g}, N \backslash F)$, that are closed under the relative equivariant differential $D_{r e l}(\eta, \xi)=\left(D \eta,\left.\eta\right|_{N \backslash F}-D \xi\right)$, while cohomology classes in the latter are defined as follows:

Let $U \subset N$ be any open, $G$-invariant subset containing $F$. We may consider the spaces $\mathcal{A}_{U}^{\infty}(\mathfrak{g}, N)$ of equivariant differential forms with support contained in $U$, and their corresponding cohomology spaces $\mathcal{H}_{U}^{\infty}(\mathfrak{g}, N)$.

If we have two open subsets $V$ and $U$ with $F \subset V \subset U$, the inclusion $\mathcal{A}_{V}^{\infty}(\mathfrak{g}, N) \hookrightarrow$ $\mathcal{A}_{U}^{\infty}(\mathfrak{g}, N)$ induces a map

$$
f_{U, V}: \mathcal{H}_{V}^{\infty}(\mathfrak{g}, N) \rightarrow \mathcal{H}_{U}^{\infty}(\mathfrak{g}, N),
$$

and so we obtain the inverse system $\left(\mathcal{H}_{U}^{\infty}(\mathfrak{g}, N), f_{U, V}, U, V \in \mathcal{F}_{F}\right)$, where $\mathcal{F}_{F}$ is the family of all open, $G$-invariant neighbourhoods of $F$, letting us define the space of cohomology with support contained in $F$ as the inverse limit of this system. 
By [12], all of the above can be extended to equivariant cohomology with generalized coefficients, including the morphism

$$
p_{F}: \mathcal{H}^{ \pm \infty}(\mathfrak{g}, N, N \backslash F) \rightarrow \mathcal{H}_{F}^{ \pm \infty}(\mathfrak{g}, N)
$$

defined in [11] as follows:

Let $U$ be any open, $G$-invariant neighbourhood of $F$, and choose a cutoff function $\chi \in C^{\infty}(N)^{G}$ with support contained in $U$, such that $\chi \equiv 1$ on a smaller neighbourhood of $F$. Let $(\eta, \xi)$ represent a class in $\mathcal{H}^{ \pm \infty}(\mathfrak{g}, N, N \backslash F)$ (so that $\left.\eta\right|_{N \backslash F}=D \xi$ ), and set

$$
p^{\chi}(\eta, \xi)=\chi \eta+d \chi \xi
$$

By Proposition 3.14 in [11], $p^{\chi}(\eta, \xi)$ is an equivariantly closed form with support in $U$, whose class $p_{U}(\eta, \xi)$ in $\mathcal{H}_{U}^{ \pm \infty}(\mathfrak{g}, N)$ does not depend on $\chi$. Moreover, $f_{U, V} \circ p_{V}=$ $p_{U}$, so that we may define $p_{F}(\eta, \xi)$ to be the element defined by taking the inverse limit over invariant neighbourhoods of $F$.

Remark 5.4. An element of $\mathcal{H}_{F}^{ \pm \infty}(\mathfrak{g}, N)$ in the image of $p_{F}$ may be represented in computations by one of the forms $p^{\chi}(\eta, \xi)$.

If $F$ is compact, there is a natural map

$$
\mathcal{H}_{F}^{ \pm \infty}(\mathfrak{g}, N) \rightarrow \mathcal{H}_{c}^{ \pm \infty}(\mathfrak{g}, N) .
$$

The composition of $p_{F}$ with (20) defines a map denoted $p_{c}$ in [11].

In the case where $N$ is a $G$-equivariant vector bundle we introduce two other complexes of differential forms: the complexes $\mathcal{A}_{r d m}^{ \pm \infty}(\mathfrak{g}, N)$ of differential forms that are rapidly decreasing in mean:

Definition 5.5. Suppose $N \rightarrow B$ is a G-equivariant vector bundle over the compact base $B$, and suppose $\beta: \mathfrak{g} \rightarrow N$ is an equivariant differential form on $N$ (possibly with generalized coefficients). We say that $\beta$ is rapidly decreasing in mean if for any smooth, compactly supported density $\rho(X)$ on $\mathfrak{g}$, the differential form $\beta_{\rho}=$ $\int_{\mathfrak{g}} \beta(X) \rho(X) d X$ and all its derivatives are rapidly decreasing along the fibres of $N \rightarrow$ $B$.

The equivariant differential $D$ is well-defined on $\mathcal{A}_{r d m}^{-\infty}(\mathfrak{g}, N)$, and so we may define the cohomology space $\mathcal{H}_{r d m}^{-\infty}(\mathfrak{g}, N)$.

Note that we have the inclusions

$$
\mathcal{A}_{r d m}^{\infty}(\mathfrak{g}, N) \hookrightarrow \mathcal{A}_{r d m}^{-\infty}(\mathfrak{g}, N) \hookleftarrow \mathcal{A}_{c}^{-\infty}(\mathfrak{g}, N)
$$

5.3. Chern characters. Suppose $\mathcal{E}=\mathcal{E}^{+} \oplus \mathcal{E}^{-}$is a $\mathbb{Z}_{2}$-graded $G$-equivariant vector bundle over $N$, and let $\mathbb{A}$ be a $G$-invariant superconnection on $\mathcal{E}$, in the sense of Quillen [13]; see also [10] or [2]. Thus $\mathbb{A}$ is an odd invariant operator on $\mathcal{A}(N, \mathcal{E})$ which preserves the $\mathbb{Z}_{2}$-grading of $\mathcal{E}$, and satisfies the derivation property

$$
\mathbb{A}(\omega s)=d \omega s+(-1)^{\operatorname{deg} \omega} \omega \mathbb{A} s,
$$

for any form $\omega$ on $N$, and any section $s$ of $\mathcal{E}$. One example is the superconnection $\mathbb{A}=\nabla+L$ on $\mathcal{E}$ considered in [10], where $\nabla$ is a $G$-invariant connection on $\mathcal{E}$ in the usual sense, and $L$ is an odd $G$-invariant endomorphism of $\mathcal{E}$. 
Definition 5.6 $([2,12])$. Given a $G$-invariant superconnection $\mathbb{A}$ on $\mathcal{E}$, we define the moment of $\mathbb{A}$ to be the map $\mu^{\mathbb{A}}: \mathfrak{g} \rightarrow \mathcal{A}(N, \operatorname{End}(\mathcal{E}))^{+}$given by

$$
\mu^{\mathbb{A}}(X)=\mathcal{L}(X)-\left[\iota_{X_{N}}, \mathbb{A}\right],
$$

where $\mathcal{L}(X)=\left[d, \iota_{X_{N}}\right]$ is the Lie derivative in the direction of $X_{N}$.

In the case $\mathbb{A}=\nabla+L$ mentioned above, the moment of $\mathbb{A}$ becomes simply $\mu(X)=$ $\mathcal{L}(X)-\nabla_{X}$.

The equivariant curvature of $\mathbb{A}$ is the map $\mathbb{F}(\mathbb{A}): \mathfrak{g} \rightarrow \mathcal{A}(N, \operatorname{End}(\mathcal{E}))^{+}$given by $\mathbb{F}(\mathbb{A})(X)=\mathbb{A}^{2}+\mu^{\mathbb{A}}(X)$, and the equivariant Chern character of $(\mathcal{E}, \mathbb{A})$ is the equivariant differential form $\operatorname{Ch}(\mathbb{A}, X)=\operatorname{Str}\left(e^{\mathbb{F}(\mathbb{A})(X)}\right)$.

The equivariant Chern character is equivariantly closed, so that $\operatorname{Ch}(\mathbb{A}, X)$ defines a class in $\mathcal{H}^{\infty}(\mathfrak{g}, N)$ equal to the Chern character of $\mathcal{E}$.

Now, if we are given a smooth, $G$-equivariant morphism $\sigma: \mathcal{E}^{+} \rightarrow \mathcal{E}^{-}$, define $\sigma^{*}$ using an invariant Hermitian metric on $\mathcal{E}$. Then the map

$$
v_{\sigma}=\left(\begin{array}{cc}
0 & \sigma^{*} \\
\sigma & 0
\end{array}\right)
$$

defines an odd Hermitian endomorphism of $\mathcal{E}$, and we can associate to it a differential form given by

$$
\operatorname{Ch}\left(\mathbb{A}^{\sigma}, X\right)=\operatorname{Ch}(\mathbb{A}(\sigma, 1), X)=\operatorname{Str}\left(e^{\mathbb{F}(\mathbb{A}, \sigma, 1)(X)}\right),
$$

where $\mathbb{A}(\sigma, t)=\mathbb{A}+i t v_{\sigma}$, and $\mathbb{F}(\mathbb{A}, \sigma, t)(X)$ is the equivariant curvature of $\mathbb{A}(\sigma, t)$. Explicitly, we have

$$
\mathbb{F}(\mathbb{A}, \sigma, t)(X)=-t^{2} v_{\sigma}^{2}+i t\left[\mathbb{A}, v_{\sigma}\right]+\mathbb{F}(\mathbb{A})(X) .
$$

We remark that in the non-equivariant setting, the Chern character (22) is essentially the form considered by Mathai-Quillen [10], in the case where $N$ is a vector bundle. We will denote by $\mathrm{Ch}_{M Q}(\sigma, X)$ the corresponding equivariant Chern character studied in [11].

If we define as well the transgression form $\eta(\mathbb{A}, \sigma, t)=-i \operatorname{Str}\left(v_{\sigma} e^{\mathbb{F}(\mathbb{A}, \sigma, t)}\right)$, then on $N \backslash \operatorname{Supp}(\sigma)$, we have the well-defined equivariant differential form $\beta(\mathbb{A}, \sigma) \in$ $\mathcal{A}^{\infty}(\mathfrak{g}, N \backslash \operatorname{Supp}(\sigma))$ given by

$$
\beta(\mathbb{A}, \sigma)=\int_{0}^{\infty} \eta(\mathbb{A}, \sigma, t) d t .
$$

Then $\left.\operatorname{Ch}(\mathbb{A})\right|_{N \backslash \operatorname{Supp}(\sigma)}=D \beta(\mathbb{A}, \sigma)[11]$, so that

$$
(\operatorname{Ch}(\mathbb{A}), \beta(\mathbb{A}, \sigma)) \in \mathcal{H}^{\infty}(\mathfrak{g}, N, N \backslash \operatorname{Supp}(\sigma)) .
$$

This is the relative Chern character $\mathrm{Ch}_{r e l}(\sigma, X)$ of [11]. With $F=\operatorname{Supp}(\sigma)$, we can use the map (18) to obtain a class

$$
\mathrm{Ch}_{\text {sup }}(\sigma, X)=p_{F}\left(\mathrm{Ch}_{\text {rel }}(\sigma, X)\right) \in \mathcal{H}_{\operatorname{Supp}(\sigma)}^{\infty}(\mathfrak{g}, N),
$$

which is independent of the superconnection $\mathbb{A}$, and can be represented in computations by an equivariant form

$$
c(\sigma, \mathbb{A}, \chi)=\chi \operatorname{Ch}(\mathbb{A})+d \chi \beta(\mathbb{A}, \sigma),
$$


where $\chi \in C^{\infty}(N)$ is a $G$-invariant cutoff function equal to 1 on a neighbourhood of $\operatorname{Supp}(\sigma)$, with support contained in $U$, for some $G$-invariant neighbourhood of $\operatorname{Supp}(\sigma)$.

Remark 5.7. If $\sigma$ is elliptic, so that $\operatorname{Supp}(\sigma)$ is compact, then we obtain a class $\mathrm{Ch}_{c}(\sigma) \in \mathcal{H}_{c}^{\infty}(\mathfrak{g}, N)$ under the the natural map (20) given in the previous subsection.

Furthermore, we have the following theorem [12]:

Theorem 5.8. Suppose $N$ is G-equivariant vector bundle over a manifold $B$. Then if $\sigma$ satisfies suitable growth conditions along the fibres of $N$ (see [12]), the form $\mathrm{Ch}_{M Q}(\sigma, X)$ is an element of $\mathcal{A}_{r d m}^{\infty}(\mathfrak{g}, N)$ and represents the image of the class $\mathrm{Ch}_{\text {sup }}(\sigma, X) \in \mathcal{H}_{\operatorname{Supp}(\sigma)}^{\infty}(\mathfrak{g}, N)$ in $\mathcal{H}_{r d m}^{\infty}(\mathfrak{g}, N)$.

Moreover, if the fibres of $\pi: N \rightarrow B$ are oriented, and the action of $G$ preserves the orientation, then we have $\pi_{*} \mathrm{Ch}_{M Q}(\sigma, X)=\pi_{*} \mathrm{Ch}_{\text {sup }}(\sigma, X)$ in $\mathcal{H}^{\infty}(\mathfrak{g}, B)$.

We now move from forms with smooth coefficients to those with generalized coefficients, which will allow us to shrink the support of our Chern character by using a $G$-invariant 1-form to modify the superconnection.

In [12] we see that the above results carry over to equivariant cohomology with generalized coefficients.

Let $\lambda \in \mathcal{A}^{1}(\mathfrak{g}, N)$ be a $G$-invariant 1-form. We use $\lambda$ to deform the part of our superconnection of exterior degree one, obtaining a new superconnection $\mathbb{A}^{\sigma, \lambda}=\mathbb{A}(\sigma, \lambda, 1)$, according to

$$
\mathbb{A}(\sigma, \lambda, t)=\mathbb{A}+i t(\sigma+\lambda), \text { for } t \in \mathbb{R} \text { and } v_{\sigma}+\lambda=\left(\begin{array}{cc}
\lambda & \sigma \\
\sigma^{*} & \lambda
\end{array}\right) .
$$

As before, we set $\mathbb{F}(\mathbb{A}, \sigma, \lambda, t)=\left(\mathbb{A}+i t\left(v_{\sigma}+\lambda\right)\right)^{2}+\mu^{\mathbb{A}}$, so that $\mathbb{F}(\mathbb{A}, \sigma, \lambda, 1)$ is the equivariant curvature of $\mathbb{A}^{\sigma, \lambda}, \operatorname{Ch}\left(\mathbb{A}^{\sigma, \lambda}\right)=\operatorname{Str}\left(e^{\mathbb{F}(\mathbb{A}, \sigma, \lambda, 1)}\right)$ is the associated character form, and

$$
\eta(\sigma, \lambda, \mathbb{A}, t)=-i \operatorname{Str}\left(\left(v_{\sigma}+\lambda\right) e^{\mathbb{F}(\mathbb{A}, \sigma, \lambda, t)}\right)
$$

the transgression form.

Then we may define $\beta(\mathbb{A}, \sigma, \lambda)=\int_{0}^{\infty} \eta(\mathbb{A}, \sigma, \lambda, t) d t$, which is now well-defined on $N \backslash\left(\operatorname{Supp}(\sigma) \cap C_{\lambda}\right)$, but only as a differential form with generalized coefficients [12].

We thus obtain a class

$$
\operatorname{Ch}_{\text {rel }}(\sigma, \lambda)=(\operatorname{Ch}(\mathbb{A}), \beta(\mathbb{A}, \sigma, \lambda)) \in \mathcal{H}^{-\infty}\left(\mathfrak{g}, N, N \backslash\left(\operatorname{Supp}(\sigma) \cap C_{\lambda}\right)\right),
$$

giving us

$$
\mathrm{Ch}_{\text {sup }}(\sigma, \lambda)=p_{F}\left(\mathrm{Ch}_{r e l}(\sigma, \lambda)\right) \in \mathcal{H}^{-\infty}(\mathfrak{g}, N, N \backslash F),
$$

where $F=\operatorname{Supp}(\sigma) \cap C_{\lambda}$. The class $\operatorname{Ch}_{\text {sup }}(\sigma, \lambda)$ is independent of $\mathbb{A}$, and can be represented by a differential form

$$
c(\sigma, \lambda, \mathbb{A}, \chi)=\chi \operatorname{Ch}(\mathbb{A})+d \chi \beta(\sigma, \lambda, \mathbb{A}),
$$

where $\chi \in C^{\infty}(N)^{G}$ is equal to 1 on a neighbourhood of $\operatorname{Supp}(\sigma) \cap C_{\lambda}$, and has support contained in a $G$-invariant neighbourhood $U$ of $\operatorname{Supp}(\sigma) \cap C_{\lambda}$.

As in the smooth case, we have the following [12]: 
Theorem 5.9. Suppose that $N$ is a $G$-equivariant vector bundle over a $G$-manifold $B$. If $\sigma$ and $\lambda$ satisfy suitable growth conditions along the fibres of $N \rightarrow B$, then $\operatorname{Ch}\left(\mathbb{A}^{\sigma, \lambda}\right) \in \mathcal{A}_{r d m}^{\infty}(\mathfrak{g}, N)$ and $\beta(\mathbb{A}, \sigma, \lambda) \in \mathcal{A}_{r d m}^{-\infty}\left(\mathfrak{g}, N \backslash\left(\operatorname{Supp}\left(\sigma \cap C_{\lambda}\right)\right)\right.$, and $\operatorname{Ch}\left(\mathbb{A}^{\sigma, \lambda}\right)$ represents the image of $\mathrm{Ch}_{\text {sup }}(\sigma, \lambda)$ in $\mathcal{H}_{r d m}^{-\infty}(\mathfrak{g}, N)$.

Moreover, if the fibres of $\pi: N \rightarrow B$ are oriented, and the action of $G$ preserves the orientation, then the morphism $\pi_{*}: \mathcal{H}_{r d m}^{-\infty}(\mathfrak{g}, N) \rightarrow \mathcal{H}^{-\infty}(\mathfrak{g}, B)$ is well-defined, and $\pi_{*} \mathrm{Ch}\left(\mathbb{A}^{\sigma, \lambda}\right)=\pi_{*} \mathrm{Ch}_{\text {sup }}(\sigma, \lambda)$ in $\mathcal{H}^{-\infty}(\mathfrak{g}, B)$.

In the case of a trivial bundle, we can define a class $P_{\kappa} \in \mathcal{H}_{C_{\kappa}}^{-\infty}(\mathfrak{g}, N)$ via $P_{\kappa}=$ $p_{F}(2 \pi, \beta(\kappa))$, where $F=C_{\kappa}$ and $\beta(\kappa)=-i \kappa \int_{0}^{\infty} e^{i t D \kappa} d t=-2 \pi i \kappa \delta_{+}(D \kappa)$, so that $D \beta=2 \pi$ by (13). If $U$ is a $G$-invariant neighbourhood of $C_{\kappa}$ and $\chi \in C^{\infty}(U)^{G}$ is equal to 1 on a neighbourhood of $C_{\kappa}$, then $P_{\kappa}$ can be represented by the form $P_{U}(\kappa, \chi)=2 \pi \chi+d \chi \beta(\kappa)$.

5.4. The case of a contact manifold. We return now to the case of a compact, co-oriented contact manifold $(M, E)$. Consider the complex vector bundle $p: E^{0,1} \rightarrow$ $M$ obtained from a $G$-invariant almost-CR structure on $M$. Equip $E^{0,1}$ with a $G$ invariant Hermitian metric $h$ compatible with the symplectic structure on $E$ and the almost-CR structure. Let $\nabla$ be a $G$-invariant Hermitian connection on $E^{0,1}$ and let $F(X)$ be its equivariant curvature.

The symbol $\sigma_{b}(10)$ on $\pi_{M}^{*} \mathcal{E}$ is just the pullback by $q: T^{*} M \rightarrow E^{*}$ of the equivariant morphism $\sigma_{E^{0,1}}^{\mathbb{C}}: p^{*}\left(\bigwedge E^{0, \text { even }}\right) \rightarrow p^{*}\left(\bigwedge E^{0, \text { odd }}\right)$ defined in [11]. Furthermore, we have $\sigma_{b}^{*}=\sigma_{b}$ with respect to the metric $h$, so that $v_{\sigma_{b}}^{2}=\sigma_{b}^{2} \mathrm{Id}$, giving $\operatorname{Ch}_{M Q}\left(\sigma_{b}, X\right)$ "Gaussian shape" along the fibres of $E^{*}$ as in [10].

If we define the equivariant Todd form of $\left(E^{0,1}, \nabla\right)$ for $X \in \mathfrak{g}$ sufficiently small by

$$
\operatorname{Td}\left(E^{0,1}, X\right)=\operatorname{det}_{\mathbb{C}}\left(\frac{F(X)}{e^{F(X)}-1}\right)
$$

then we have [11]

$$
\mathrm{Ch}_{M Q}\left(\sigma_{E^{0,1}}^{\mathbb{C}}, X\right)=(2 \pi i)^{n} p^{*}\left(\operatorname{Td}\left(E^{0,1}, X\right)^{-1}\right) \operatorname{Th}_{M Q}\left(E^{0,1}\right) \text { in } \mathcal{H}_{r d m}^{\infty}\left(\mathfrak{g}, E^{0,1}\right),
$$

where $\operatorname{Th}_{M Q}\left(E^{0,1}\right)$ is an equivariant version of the Thom form defined in [10], and $n$ is the complex rank of $E^{0,1}$.

If we pull back the above result to $T^{*} M$, then we obtain

$$
\mathrm{Ch}_{M Q}\left(\sigma_{b}, X\right)=(2 \pi i)^{n} \pi_{M}^{*}\left(\operatorname{Td}\left(E^{0,1}, X\right)^{-1}\right) q^{*}\left(\operatorname{Th}_{M Q}\left(E^{0,1}\right)\right),
$$

where $\operatorname{Ch}_{M Q}\left(\sigma_{b}, X\right)=\operatorname{Ch}\left(\mathbb{A}_{b}^{\sigma}, X\right)$.

Let $\theta$ be the canonical 1-form on $T^{*} M$. Since the action of $G$ on $M$ is assumed to be elliptic, we know that $F=\operatorname{Supp}\left(\sigma_{b}\right) \cap C_{\theta}=T_{G}^{*} M \cap E^{0}=\{0\}$ is compact. Thus $\mathrm{Ch}_{\text {sup }}\left(\sigma_{b}, \theta\right)$ defines a class in $\mathcal{H}_{c}^{-\infty}\left(\mathfrak{g}, T^{*} M\right)$ under the mapping $(20)$.

Denote by $\mathrm{Ch}_{B V}\left(\sigma_{b}, X\right)$ the Chern character of [3], given by

$$
\mathrm{Ch}_{B V}\left(\sigma_{b}, X\right)=\operatorname{Str}\left(e^{F\left(\mathbb{A}, \sigma_{b}, \theta, 1\right)(X)}\right),
$$

which is an element of $\mathcal{A}_{r d m}^{\infty}\left(\mathfrak{g}, T^{*} M\right)$.

By Theorem 5.9, the images of $\mathrm{Ch}_{B V}\left(\sigma_{b}, X\right)$ and $\mathrm{Ch}_{\text {sup }}\left(\sigma_{b}, \theta\right)$ under the maps induced by the inclusions (21) coincide in $\mathcal{H}_{r d m}^{-\infty}\left(\mathfrak{g}, T^{*} M\right)$. 
Similarly, by Theorem 5.8, a representative of $\mathrm{Ch}_{\text {sup }}\left(\sigma_{b}\right)$ in $\mathcal{H}_{r d m}^{\infty}\left(\mathfrak{g}, T^{*} M\right)$, is given by $\mathrm{Ch}_{M Q}\left(\sigma_{b}, X\right)$, which we relate to $\mathrm{Ch}_{B V}\left(\sigma_{b}, X\right)$ using following two lemmas from [12]:

Lemma 5.10. Let $\kappa$ be a $G$-invariant 1-form on $N$, and define $P_{\kappa}$ as above. Then:

(1) Under the natural map $\mathcal{H}_{C_{\kappa}}^{-\infty}(\mathfrak{g}, N) \rightarrow \mathcal{H}^{-\infty}(\mathfrak{g}, N)$, the image of $P_{\kappa}$ is equal to 1 .

(2) $\operatorname{Ch}(\sigma, \kappa)=P_{\kappa} \wedge \operatorname{Ch}(\sigma)$ in $\mathcal{H}_{\operatorname{Supp}(\sigma) \cap C_{\kappa}}^{-\infty}(\mathfrak{g}, N)$.

Lemma 5.11. If $\left.\theta\right|_{\operatorname{Supp}(\sigma)}=\left.\lambda\right|_{\operatorname{Supp}(\sigma)}$, then $\operatorname{Supp}(\sigma) \cap C_{\theta}=\operatorname{Supp}(\sigma) \cap C_{\lambda}=T_{G}^{*} M \cap$ $E^{0}$, and $\operatorname{Ch}(\sigma, \theta)=\operatorname{Ch}(\sigma, \lambda)$ in $\mathcal{H}_{T_{G}^{*} M \cap E^{0}}^{-\infty}\left(\mathfrak{g}, T^{*} M\right)$.

Together, the two above lemmas give:

Proposition 5.12. Let $i: E^{0} \hookrightarrow T^{*} M$ be the inclusion of $E^{0}$, and define $\lambda=i^{*} \theta$. Using the splitting $T^{*} M=E^{*} \oplus E^{0}$, consider $\lambda$ as a form on all of $T^{*} M$, by taking $\left.\lambda\right|_{E^{*}}=0$, and $\left.\lambda\right|_{E^{0}}=i^{*} \theta$.

Then, $\lambda$ and $\theta$ agree on $\operatorname{Supp}\left(\sigma_{b}\right)=E^{0}$, and we have

$$
\mathrm{Ch}_{B V}\left(\sigma_{b}, X\right)=P_{\lambda}(X) \wedge \mathrm{Ch}_{M Q}\left(\sigma_{b}, X\right) \text { in } \mathcal{H}_{r d m}^{-\infty}\left(\mathfrak{g}, T^{*} M\right) .
$$

\section{Calculation of the index}

We now apply the results of the previous section, in the case of a compact, cooriented contact manifold $(M, E)$ to the Berline-Vergne index formula (3).

Recall that the equivariant $\hat{A}$-class is defined for any real $G$-equivariant vector bundle $\mathcal{E} \rightarrow M$, with $G$-equivariant connection $\nabla$ and corresponding equivariant curvature $F(X)$ by

$$
\hat{A}(\mathcal{E}, X)=\operatorname{det}_{\mathbb{R}}^{1 / 2}\left(\frac{F(X)}{e^{F(X) / 2}-e^{-F(X) / 2}}\right),
$$

with the choice of square root depending on orientation. The equivariant $\hat{A}$-class of $T M \rightarrow M$ is denoted by $\hat{A}(M, X)$.

The form $D_{\mathbb{R}}(N(g), X)$ associated to the normal bundle is defined in [3] as follows:

Definition 6.1. For $g \in G$, let $F_{N}(X), X \in \mathfrak{g}(g)$, denote the equivariant curvature of $N(g)$ with respect to a $G(g)$-equivariant connection. Then $D_{\mathbb{R}}(N(g), X)$ is the $G(g)$-equivariantly closed from on $M(g)$ given for $X \in \mathfrak{g}(g)$ by

$$
D_{\mathbb{R}}(N(g), X)=\operatorname{det}_{\mathbb{R}}\left(1-g^{N} e^{F_{N}(X)}\right),
$$

where $g^{N}$ denotes the lifted action of $g \in G$ on $N(g)$.

We similarly define $D_{\mathbb{C}}(N(g), X)$ using the complex determinant in place of the real determinant used above. Note that using the complex structure on $N(g)$ induced from that on $E(g)$, we may write $N(g) \otimes \mathbb{C}=N(g) \oplus \overline{N(g)}$ and obtain:

$$
D_{\mathbb{R}}(N(g), X)=D_{\mathbb{C}}(N(g) \otimes \mathbb{C}, X)=D_{\mathbb{C}}(N(g), X) D_{\mathbb{C}}(\overline{N(g)}, X) .
$$

We are now ready to state the main theorem of this article: 
Theorem 6.2. Let $(M, E)$ be a compact, co-oriented contact manifold of dimension $2 n+1$, and let $G$ be a compact Lie group acting elliptically on $M$. Let $g \in G$, and let $k(g)$ be the locally constant function defined by $\operatorname{dim} M(g)=2 k(g)+1$.

The $G$-equivariant index of $D_{b}$ is the generalized function on $G$ whose germ at $g \in G$, is given, for $X \in \mathfrak{g}(g)$ sufficiently small, by

$$
\operatorname{index}^{G}\left(\not_{b}\right)\left(g e^{X}\right)=\int_{M(g)}(2 \pi i)^{-k(g)} \frac{\operatorname{Td}(E(g), X) \mathcal{J}(E(g), X)}{D_{\mathbb{C}}(N(g), X)} .
$$

6.1. The formula near the identity. We first consider the index formula for group elements $e^{X}$, for $X \in \mathfrak{g}$ sufficiently small. The calculation in this case is simpler, and employs the results of [12] directly. The general result will then follow an analogous approach.

Theorem 6.3. For $X \in \mathfrak{g}$ sufficiently small, we have

$$
\operatorname{index}^{G}\left(\emptyset_{b}\right)\left(e^{X}\right)=\frac{1}{(2 \pi i)^{n}} \int_{M} \operatorname{Td}(E, X) \mathcal{J}(M, X) \text {. }
$$

Proof. The formula of Berline-Vergne for the equivariant index of a transversally elliptic operator is given by

$$
\operatorname{index}^{G}\left(\not_{b}\right)\left(e^{X}\right)=\frac{1}{(2 \pi i)^{(2 n+1)}} \int_{T^{*} M} \pi_{M}^{*}\left(\hat{A}^{2}(M, X)\right) \operatorname{Ch}_{B V}\left(\sigma_{b}, X\right) .
$$

Using the splitting $T M=E \oplus \mathbb{R}$, and the almost-complex structure on $E$, we have that

$$
\hat{A}^{2}(M, X)=\hat{A}^{2}(E, X)=\operatorname{Td}(E \otimes \mathbb{C}, X)=\operatorname{Td}\left(E^{1,0}, X\right) \operatorname{Td}\left(E^{0,1}, X\right) .
$$

By (26) and (27), we have

$$
\begin{aligned}
\mathrm{Ch}_{B V}\left(\sigma_{b}, X\right) & =P_{\lambda}(X) \mathrm{Ch}_{M Q}\left(\sigma_{b}, X\right) \\
& =(2 \pi i)^{n} P_{\lambda}(X) \pi_{M}^{*}\left(\operatorname{Td}\left(E^{0,1}, X\right)^{-1}\right) q^{*}\left(\operatorname{Th}_{M Q}\left(E^{0,1}\right)\right) .
\end{aligned}
$$

Combining (31) and (32), we find

$$
\begin{aligned}
& \pi_{M}^{*}\left(\hat{A}^{2}(M, X)\right) \mathrm{Ch}_{B V}\left(\sigma_{b}, X\right)= \\
& (2 \pi i)^{n} \pi_{M}^{*}\left(\operatorname{Td}\left(E^{1,0}, X\right)^{-1}\right) q^{*}\left(\operatorname{Th}_{M Q}\left(E^{0,1}\right)\right) P_{\lambda}(X)
\end{aligned}
$$

in $\mathcal{H}_{r d m}^{-\infty}\left(\mathfrak{g}, T^{*} M\right)$.

Lemma 6.4. In terms of the projections $q: T^{*} M \rightarrow E^{*}$ and $p: E^{*} \rightarrow M$ we have

$$
q_{*} P_{\lambda}(X)=2 \pi i p^{*} \mathcal{J}(E, X) .
$$

That is, $\left(\pi_{M}\right)_{*} P_{\lambda}=p_{*} q_{*} P_{\lambda}=2 \pi i \mathcal{J}(E, X)$ in $\mathcal{H}^{-\infty}(\mathfrak{g}, M)$. 
Proof. A representative of $P_{\lambda}$ is given by

$$
P_{\lambda}^{\chi}=2 \pi \chi+d \chi \wedge \beta(\lambda)=2 \pi \chi-2 \pi i d \chi \wedge \lambda \delta_{+}(D \lambda),
$$

where $\chi$ is a cutoff function with support in a neighbourhood of $E^{*}$, and $\chi \equiv 1$, on $E^{*}$.

Since $\lambda$ is a form on $E^{0}$, and $\chi$ is constant on $E^{*}, P_{\lambda}$ is independent of $E^{*}$, and so it remains to calculate the integral over the fibre of $E^{0}=M \times \mathbb{R}$. Let $t$ be the co-ordinate along the fibre, and write $\chi=\chi(t)$. Then $\chi(t)$ is supported on a neighbourhood of $t=0$, with $\chi(0)=1$, and $\lambda$ may be written as $\lambda=-t \alpha$, for $\alpha$ a contact form on $M$. Thus $D \lambda=D(-t \alpha)=\alpha \wedge d t-t D \alpha$, and $P_{\lambda}$ becomes

$$
\begin{aligned}
P_{\lambda} & =2 \pi \chi(t)-2 \pi i \chi^{\prime}(t) d t \wedge(-t \alpha) \delta_{+}(\alpha \wedge d t-t D \alpha) \\
& =2 \pi \chi(t)-2 \pi i \alpha \wedge t \chi^{\prime}(t) d t \delta_{+}(-t D \alpha) .
\end{aligned}
$$

Thus, the integral over $\mathbb{R}$ becomes, with the help of the identities in Section 5.1,

$$
\begin{aligned}
\int_{-\infty}^{\infty} P_{\lambda} & =-2 \pi i \alpha \int_{-\infty}^{\infty} \chi^{\prime}(t) t \delta_{+}(-t D \alpha) d t \\
& =-2 \pi i \alpha\left[\int_{0}^{\infty} \chi^{\prime}(t) \delta_{-}(D \alpha) d t-\int_{-\infty}^{0} \chi^{\prime}(t) \delta_{+}(D \alpha) d t\right] \\
& =-2 \pi i \alpha\left[-\delta_{-}(D \alpha)-\delta_{+}(D \alpha)\right] \\
& =2 \pi i \alpha \delta_{0}(D \alpha),
\end{aligned}
$$

and we obtain our result.

Let $\operatorname{Td}(E, X)$ denote the cohomology class of the Todd form $\operatorname{Td}\left(E^{1,0}, X\right)$, and write $\operatorname{Th}_{M Q}\left(E^{0,1}\right)=\operatorname{Th}_{M Q}\left(E^{*}\right)$ using the isomorphism (7). Then using Lemma 6.4 and (33) in the index formula (30), we obtain

$$
\begin{aligned}
\operatorname{index}^{G}\left(\not D_{b}\right)\left(e^{X}\right) & =(2 \pi i)^{-(2 n+1)} \int_{T^{*} M}(2 \pi i)^{n} \pi_{M}^{*}(\operatorname{Td}(E, X)) q^{*} \operatorname{Th}_{M Q}\left(E^{*}\right) P_{\lambda}(X) \\
& =\frac{1}{(2 \pi i)^{n}} \int_{E^{*}} p^{*}(\operatorname{Td}(E, X) \mathcal{J}(E, X)) \operatorname{Th}_{M Q}\left(E^{*}\right) \\
& =\frac{1}{(2 \pi i)^{n}} \int_{M} \operatorname{Td}(E, X) \mathcal{J}(E, X)
\end{aligned}
$$

6.2. Fixed Point Formula. The general calculation of the push-forward of (3) from $T^{*} M(g)$ to $M(g)$ is analogous to the proof given above, since by Proposition 2.7, we know that $(M(g), E(g))$ is again a contact manifold on which $G(g)$ acts elliptically. The primary added difficulty comes from the appearance of the action of $g \in G$ in the Chern character form of [3].

Proof of Theorem 6.2: By Proposition 2.7, $(M(g), E(g))$ is again a contact manifold, and we have the splitting

$$
T^{*} M(g)=E^{*}(g) \oplus \mathbb{R} \alpha^{g} .
$$


Let $\operatorname{dim} M(g)=2 k(g)+1$, so that $E(g)$ is a vector bundle over $M(g)$ of complex rank $k(g)$.

Denote by $j: T^{*} M(g) \rightarrow T^{*} M$ the inclusion of the $g$-fixed point set in $T^{*} M$. Let $H=G(g)$, with Lie algebra $\mathfrak{h}$. Let $\mathbb{A}^{g}=j^{*} \mathbb{A}, \theta^{g}=j^{*} \theta$ and $\sigma_{b}^{g}=j^{*} \sigma_{b}$ denote the restrictions of the superconnection, canonical 1-form and symbol of the previous section to $T^{*} M(g)$. Let $p, q$ denote the projections $p: E^{*}(g) \rightarrow M(g)$ and $q$ : $T^{*} M(g) \rightarrow E^{*}(g)$.

The Chern character $\mathrm{Ch}_{B V}^{g}\left(\sigma_{b}\right)(X)$ of Berline and Vergne is given by

$$
\mathrm{Ch}_{B V}^{g}\left(\sigma_{b}\right)(X)=\operatorname{Str}\left(g^{\mathcal{E}} \cdot j^{*}\left(e^{\mathbb{F}\left(\mathbb{A}, \sigma_{b}, \theta\right)(X)}\right)\right) .
$$

By [3], since $\sigma_{b}$ is $G$-transversally elliptic, $\sigma_{b}^{g}$ is $H$-transversally elliptic, and $\mathrm{Ch}_{B V}^{g}\left(\sigma_{b}\right)$ defines a class in $\mathcal{H}_{r d m}^{\infty}\left(\mathfrak{h}, T^{*} M(g)\right)$.

Let $j^{*} \nabla=\nabla_{E} \oplus \nabla_{N}$ denote the decomposition of the restriction of the invariant Hermitian connection $\nabla$ on $E^{0,1}$ into connections on $E^{0,1}(g)$ and $N(g)$, respectively. Since $\mathbb{A}=q^{*} \nabla$, we have $\mathbb{A}^{g}=\mathbb{A}_{E} \oplus \nabla_{N}$, where $\mathbb{A}_{E}=q^{*} \nabla_{E}$.

By Lemma 19 of [3], the canonical 1-form on $T^{*} M(g)$ is simply the restriction $\theta^{g}$ of the canonical 1-form $\theta$ on $T^{*} M$. Since $\theta^{g}$ is invariant under the action of $g$, we have

$$
\mathrm{Ch}_{B V}^{g}\left(\sigma_{b}, X\right)=e^{i D \theta^{g}} \operatorname{Str}\left(g^{\mathcal{E}} \cdot e^{\mathbb{F}\left(\mathbb{A}_{E} \oplus \nabla_{N}, \sigma_{b}\right)(X)}\right) .
$$

Lemma 6.5. Let $V$ be a complex vector space of dimension $k$, and let a Lie group $G$ act on $V$, such that the action commutes with the natural $U(k)$ action on $V$. Let $\rho: U(k) \rightarrow \bigwedge V^{*}$ denote the representation of $U(k)$ on $\bigwedge V^{*}$ as in [10], and let $w \in \mathfrak{u}(k)$ be a skew-symmetric Hermitian matrix. Then for any $g \in G$, we have

$$
\operatorname{Str}\left(g \cdot e^{\rho(w)}\right)=\operatorname{det}_{\mathbb{C}}\left(1-g \cdot e^{-w}\right) .
$$

Proof. Since the action of $G$ on $\bigwedge V^{*}$ commutes with the representation $\rho$, the actions of $g$ and $w$ can be simultaneously diagonalized.

Using the above Lemma we may write

$$
\mathrm{Ch}_{B V}^{g}\left(\sigma_{b}, X\right)=e^{i D \theta^{g}} \operatorname{Ch}\left(\mathbb{A}_{E}, \sigma_{b}^{g}, X\right) D_{\mathbb{C}}(\bar{N}(g), X),
$$

using

$$
\operatorname{det}_{\mathbb{C}}\left(1-g^{\mathcal{E}} \cdot\left(j^{*} e^{-\mathbb{F}\left(\mathbb{A}_{E} \oplus \nabla\right)(X)}\right)\right)=\operatorname{det}_{\mathbb{C}}\left(1-e^{-\mathbb{F}\left(\mathbb{A}_{E}\right)(X)}\right) \operatorname{det}_{\mathbb{C}}\left(1-g^{N} \cdot e^{-F\left(\nabla_{N}\right)(X)}\right),
$$

since $g$ acts trivially on $T^{*} M(g)$.

The form $\operatorname{Ch}\left(\mathbb{A}_{E}, \sigma_{b}^{g}, X\right)$ appearing in (34) is simply the Mathai-Quillen form $\mathrm{Ch}_{M Q}\left(\sigma_{b}^{g}, X\right)$ on $E^{0,1}(g)$. We again use Theorem 5.9 and Lemmas 5.10 and 5.11 as follows:

The class $e^{i D \theta^{g}(X)} \mathrm{Ch}_{M Q}\left(\sigma_{b}^{g}, X\right)=\mathrm{Ch}\left(\mathbb{A}_{E}, \sigma_{b}^{g}, \theta^{g}, X\right) \in \mathcal{A}_{r d m}^{\infty}\left(\mathfrak{h}, T^{*} M(g)\right)$ is a representative of $\mathrm{Ch}_{\text {sup }}\left(\sigma_{b}^{g}, \theta^{g}, X\right) \in \mathcal{H}_{\text {supp }\left(\sigma_{b}^{g}\right) \cap C_{\theta g}}^{-\infty}\left(\mathfrak{h}, T^{*} M(g)\right)$ in $\mathcal{H}_{r d m}^{-\infty}\left(\mathfrak{h}, T^{*} M(g)\right)$, and we have

$$
\mathrm{Ch}_{\text {sup }}\left(\sigma^{g}, \theta^{g}, X\right)=\mathrm{Ch}_{\text {sup }}\left(\sigma^{g}, \lambda^{g}, X\right)=P_{\lambda^{g}}(X) \mathrm{Ch}_{\text {sup }}\left(\sigma^{g}, X\right) .
$$

Since a representative of $\mathrm{Ch}_{\text {sup }}\left(\sigma_{b}^{g}, X\right)$ is $\mathrm{Ch}_{M Q}\left(\sigma_{b}^{g}, X\right)$, we have

$$
\mathrm{Ch}_{B V}\left(\sigma_{b}, X\right)=P_{\lambda^{g}}(X) \mathrm{Ch}_{M Q}\left(\sigma_{b}^{g}, X\right) D_{\mathbb{C}}(\bar{N}(g), X)
$$


in $\mathcal{H}_{\text {supp }\left(\sigma_{b}^{g}\right) \cap C_{\theta g}}^{-\infty}\left(\mathfrak{h}, T^{*} M(g)\right)$.

As before, $\mathrm{Ch}_{M Q}^{g}\left(\sigma_{b}\right)$ is the pull-back of a form on $E^{*}(g)$, while $P_{\lambda^{g}}$ depends only on $\mathbb{R} \alpha^{g}$. Integrating $P_{\lambda^{g}}$ over $\mathbb{R}$ proceeds the same as in the proof of Theorem 6.3, giving $2 \pi i \mathcal{J}(E(g), X)$ as the result.

Finally, we substitute (35) into (3) and use (26) and Lemma 6.4 to obtain (28).

Suppose now that $(B, \omega, \Phi)$ is a Hamiltonian $G$-manifold, which is prequantizable in the sense of [7]. Let $\mathbb{L} \rightarrow B$ be a $G$-equivariant prequantum line bundle, and let $\pi: M \rightarrow B$ be the unit circle bundle inside of $\mathbb{L}$ with respect to a Hermitian metric. A $G$-equivariant prequantization of $(B, \omega, \Phi)$ is defined in [7] to be the pair $(M, \tilde{\alpha})$, where $\tilde{\alpha}$ is a connection form on $M$, such that $i D \tilde{\alpha}(X)=\pi^{*}(\omega-\Phi(X)){ }^{2}$

Let $\not$ denote the Dolbeault-Dirac operator on sections of $\bigwedge T^{0,1} B$, and let $\sigma_{m}=$ $\sigma(\not D) \otimes \operatorname{Id}_{\mathbb{L} \otimes m}$.

Since the form $\alpha=i \tilde{\alpha}$ is a contact form on $M$, and the action of $G \times U(1)$ on $M$ is elliptic with respect to $E=\operatorname{ker}(\alpha)$, our index formula in this case provides the following:

Corollary 6.6. We have the following equality of generalized functions on $G \times U(1)$ :

$$
\operatorname{index}^{G \times U(1)}\left(\not_{b}\right)(g, u)=\sum_{m \in \mathbb{Z}} u^{-m} \operatorname{index}^{G}\left(\sigma_{m}\right)(g) .
$$

Proof. With the right identifications, this result can be viewed as a special case of Théorème 25 in [4] for $H=U(1)$, and the details of the proof are similar.

We need to check that, for any fixed $(g, u) \in G \times U(1)$, the formula holds in a sufficiently small neighbourhood of $(g, u)$ in $G(g) \times U(1)$. That is, for $X \in \mathfrak{g}(g)$ and $\phi \in \mathbb{R}$ sufficiently small, we need to show that

$$
\operatorname{index}^{G \times U(1)}\left(D_{b}\right)\left(g e^{X}, u e^{i \phi}\right)=\sum_{m \in \mathbb{Z}} u^{-m} e^{-i m \phi} \operatorname{index}^{G}\left(\sigma_{m}\right)\left(g e^{X}\right) .
$$

For any $v \in U(1)$, we have $M(g, v)=\{y \in M \mid g \cdot y=y \cdot v\}$. When $M(g, v)$ is non-empty, $U(1)$ acts freely on $M(g, v)$, and we denote $B(g)^{v}=M(g, v) / U(1)$. The fixed-point set $B(g)$ is a (finite) disjoint union of the spaces $B(g)^{v}$.

Since $\mathbb{L} \cong M \times_{U(1)} \mathbb{C}$, the action of $g \in G$ on the fibres of $\left.\mathbb{L}\right|_{B(g)^{v}}$ is scalar multiplication by $v \in U(1)$. Thus, $\left.\mathrm{Ch}_{g}\left(\mathbb{L}^{\otimes m}, X\right)\right|_{B(g)^{v}}=v^{m} e^{i m \omega(X)}$, and we have

$$
\operatorname{index}^{G}\left(\sigma_{m}\right)\left(g e^{X}\right)=\sum_{\substack{v \in U(1) \\ M(g, v) \neq \emptyset}} \int_{B(g)^{v}}(2 \pi i)^{-k(g)} \frac{\operatorname{Td}\left(B(g)^{v}, X\right)}{D_{\mathbb{C}}\left(N_{B}(g), X\right)} v^{m} e^{i m \omega(X)} .
$$

Thus, the only contribution to the right-hand side of (37) comes from $B(g)^{u}$ (provided $M(g, u)$ is non-empty), in which case we can apply the Poisson summation formula to obtain

$$
\sum_{m \in \mathbb{Z}} u^{-m} e^{-i m \phi} \operatorname{index}^{G}\left(\sigma_{m}\right)\left(g e^{X}\right)=\int_{B(g)^{u}}(2 \pi i)^{-k(g)} \frac{\operatorname{Td}\left(B(g)^{u}, X\right) \delta_{0}\left(\omega^{g}(X)-\phi\right)}{D_{\mathbb{C}}\left(N_{B}(g), X\right)} .
$$

\footnotetext{
${ }^{2}$ We are using the convention here that $M$ is a principal $U(1)$-bundle, and that $\mathfrak{u}(1)=i \mathbb{R}$.
} 
Using the index formula (28), the left-hand side of (37) is given by

$$
\int_{M(g, u)}(2 \pi i)^{-k(g)} \frac{\operatorname{Td}(E(g, u),(X, \phi)) \alpha^{g, u} \delta_{0}\left(D \alpha^{g, u}(X)-\phi\right)}{D_{\mathbb{C}}\left(N_{M}(g, u),(X, \phi)\right)} .
$$

The prequantization condition implies that $D \alpha^{g, u}(X, i \phi)=\pi^{*} \omega^{g}(X)-\phi$, and since the forms $\operatorname{Td}(E(g, u))$ and $D_{\mathbb{C}}\left(N_{M}(g, u)\right)$ are the pullback to $M(g, u)$ of the corresponding forms on $B(g)^{u}$, the result follows.

Acknowledgements. Many of the ideas in this paper, including the statements of the main theorems and an outline of their proof, were first suggested by my thesis advisor, Eckhard Meinrenken. I am grateful to him for suggesting this problem, and for much helpful advice and feedback on this paper.

I would also like to thank Paul-Emile Paradan for his helpful discussion while at Utrecht in the summer of 2007, and Michele Vergne for her valuable feedback on this paper. The special case of Corollary 6.6 above was suggested by both Meinrenken and Paradan.

\section{References}

[1] M. F. Atiyah, Elliptic Operators and Compact Groups, Vol. 401 of Lecture Notes in Mathematics, Springer-Verlag, Berlin (1974). Joint work with I. Singer.

[2] N. Berline, E. Getzler, and M. Vergne, Heat Kernels and Dirac Operators, Vol. 298 of Grundlehren, Springer-Verlag, Berlin (1991).

[3] N. Berline and M. Vergne, The Chern character of a transversally elliptic symbol and the equivariant index, Invet. Math. 124 (1996) 11-49.

[4] - L'indice équivariant des opérateurs transversalement elliptiques, Invent. Math. 124 (1996) 51-101.

[5] A. Boggess, CR manifolds and the tangential Cauchy-Riemann complex, CRC Press, Boca Raton, Florida (1991).

[6] G. Friedlander and M. Joshi, Introduction to the Theory of Distributions, Cambridge University Press, Cambridge, 2nd edition (1998).

[7] V. Ginzburg, V. Guillemin, and Y. Karshon, Moment Maps, Cobordisms and Hamiltonian Group Actions, Vol. 98 of Mathematical Surveys and Monographs, American Mathematical Society (2002).

[8] S. Kumar and M. Vergne, Equivariant cohomology with generalised coefficients, Astérisque 215 (1993) 109-204.

[9] E. Lerman, Contact toric manifolds, J. Symplectic Geom. 1 (2003), no. 4, 785-828.

[10] V. Mathai and D. Quillen, Superconnections, Thom classes and equivariant differential forms, Topology 25 (1986) 85-110.

[11] P.-E. Paradan and M. Vergne, Equivariant relative Thom forms and Chern characters, preprint, arXiv:0711.3898v1 [math.DG] (2007).

[12] — Equivariant Chern character with generalized coefficients, preprint, arXiv:0801.2822v1 [math.DG] (2008).

[13] D. Quillen, Superconnections and the Chern character, Topology 24 (1985) 37-41.

[14] M. Vergne, Applications of equivariant cohomology, in International Congress of Mathematicians, Madrid, Spain, 2006, Vol. 1, 635-664, European Mathematical Society (2007).

Department of Mathematics, University of Toronto, Toronto, Ontario, Canada m5S $2 \mathrm{E} 4$

E-mail address: dfitzpat@math.toronto.edu 